\title{
Institutional Collaborative Research Training in Open and Distance Learning: A South African Phenomenological Case Study
}

\author{
Keleco Joyce Karel \\ Department of Adult Basic Education and Training, \\ University of South Africa, Pretoria, South Africa \\ Email address: karelkjn@unisa.ac.za
}

\section{Doi:10.5901/mjss.2014.v5n3p381}

\begin{abstract}
The purpose of this study was to explore and describe collaborative research training between open and distance learning $(O D L)$ institutions with the aim of increasing research output in the South African context. Data for this study were collected through the use of semi-structured interviews to understand how institutions could collaborate to increase their research outputs. Data were collected, recorded and analysed using Collaizi's data analysis framework. The framework was chosen for its ability to provide clear steps for data presentation and analysis. The study revealed that institutional collaboration is the only answer to improve research output in ODL. Furthermore, the study found out that developed researchers in various ODL institutions appear to be working in separate silos that are full of experiences but unable to share with those who are still developing. This study therefore argues that if developed and novice researchers from various ODL institutions can collaboratively work together, research output can drastically be improved.
\end{abstract}

Keywords: Open distance learning, phenomenology, collaboration, research output

\section{Introduction}

The purpose of this research study was to explore and describe institutional collaboration in research training to increase research output in an open and distance learning contexts. The challenge for all ODL institutions is to have more research output. Although there have been many previous studies of research collaboration, comparatively little attention has been given to the concept of collaboration between institutions with the aim of increasing research output in an ODL context. Collaboration in academia is becoming more common, for a variety of reasons. Academic institutions, colleges and newly established universities often seek academic alliances and collaboration with larger, more established universities to bring value, recognition and acceptance to their programs (Gibbons, 1994). According to Gibbons (1994) collaboration has been considered as one of the main characteristics of research as well as the new way of knowledge production. During collaboration, institutions share resources of any type with one or more partner institutions. Collaboration may be between institutions in the same neighbourhood or anywhere in the world. According to Hemmings (1984) collaborative relationships extend and expand the capacity of the participants to accomplish objectives that could not be accomplished by any participant alone. Institutions that are forming collaborations do not have to have similar level of expertise to collaborate, but they do need to have resources that other institutions would like to share (McCord, 2002). In the scientific and scholarly professions, a striking trend is the growth over the past several decades of collaborative work (Fox \& Faver, 1982). Research output is one of the major areas that are regarded as important in ODL because nowadays the advent of knowledge explosion is ever present. In this manner, research in ODL should drive the processes of generating new knowledge as a means of professional mobility.

\section{Literature Review}

While there is a huge literature on institutional collaboration in higher education on various topics, there is scant literature on collaborative research training in ODL institutions with the aim of increasing research output. To date, the literature on academic development has been strongly focused on the teaching aspect of academic, with collaborative research and research training being a relatively neglected area. Furthermore, there has been little collated data on the extent, nature and outcomes of ODL institutions' expenditure on collaborative research training. Therefore, it is the objective of this study to look at how ODL universities can collaboratively work together to increase research output through research 
training. Numerous authors have studied the phenomenon of collaboration, for instance, for Gray (1989) collaboration in the research context involves several concrete and symbolic meanings. Collaboration is frequently linked to formal and informal research alliances and network. Collaboration does not simply mean putting people together and expecting a better result. Furthermore, Gray (1989) defined collaboration as a process through which parties who see different aspects of a problem can explore constructively their differences and search for solution that go beyond their own limited vision of what is possible.

The term collaboration initially referred to researchers working together within the same discipline, within an institution or in different institutions. Collaboration is about structuring an arrangement for the joint provision of outputs and outcomes. In essence, collaboration is a process of interaction through which participants produce something new (Gros \& Adrian, 2005; Kitchen \& McDougall, 1999) and where particular individuals' contributions are indistinguishable from the whole (Hathorne \& Ingram, 2002). Collaboration according to Bardach $(1998,8)$ involves "joint activity by ...agencies that is intended to increase public value by their working together." This is supported by Mattessich (2001) who defines collaboration as a mutually beneficial and well relationship entered into by two or more organizations with a commitment to a set of common goals, a jointly developed structure and shared responsibility, and mutual authority and accountability. Collaboration among organizations is rapidly becoming common in research as globalisation and new communication technologies make it possible for researchers from different locations and institutions to work together on common projects.

According to Kyvik and Larsen (1997) collaborative research is the strongest form of relationship between research partners. Such collaborative research can be considered from the standpoint of either the types of structures or the types of partners involved. As for research partners, they can come from universities, industry or other institutions. However, while a wide range of factors apparently identified, few specific reasons have been established to explain how and why it occurs. Collaboration is the most formal inter organisational relationship involving shared authority and responsibility for planning, implementation, and evaluation of a joint effort. Zuckerman, Kaluzny and Rickets (1995) have described collaboration as a process that enables independent individuals and organizations to combine their human and material resources so they can accomplish objectives they are unable to bring about alone. Collaborative open distance learning institutions are believed to result in students who are more highly motivated, who learn more, and who are generally more successful (Kitchen \& McDougall, 1999). Institutional collaboration involves creating close working relationships among participants from different institutions.

Education-related collaborations can include many different activities and can take place between stakeholders at different levels. The main aims of such collaborations are to increase the students' work-relevant competencies and skills, make them more employable, foster entrepreneurial attitudes and mind sets among them, increase the flow of knowledge across sectors and stimulate the development of new networks. Institutional collaboration is sharing resources of any type with one or more partner institutions. In distance education, collaboration may be between institutions in the same neighbourhood or state, within the same country or region, and anywhere in the world. In collaboration, institutions share:

- Knowledge and expertise, for example joint research projects, designing and delivering curriculum and course material

- Infrastructure, for example information and communications technology (ICT) facilities such as video, audio conferencing equipment and computer rooms

- Human resources, for example formal arrangements for external examination or peer review

- Services, such as libraries, bookstores, student counselling

- Costs of starting or maintaining educational programmes

- Institutional culture and perspective, for example through student or faculty exchange programmes.

Institutional collaboration is understood as the research activities established by academics, departments and universities to share and gain knowledge, to increase research training and to reach common goals. In the institution environment, the term 'collaboration 'involves a wide range of possibilities, including teaching, student participation, lifelong learning, as well as commercialisation of research and intellectual property, technology transfer, consultancy and research. According to Austin and Baldwin (1992) many professors believe collaboration increases productivity, maintains motivation and stimulates creativity and risk taking. Much of the collaboration is taking place in the context of distance and distributed learning environments.

These new distributed collaborative environments would allow experts in dispersed locations to collaborate, to rapidly apply novel technologies, to create better products in less time while better managing risk, and overall to significantly facilitate the creation of new knowledge. 
Collaboration assumes importance as a means of sharing resources, learning together, cost sharing and deriving benefits from available expertise for the common good in the twenty-first century. The collaboration that is formed by open distance learning can contribute positively towards teaching and learning. Institutional collaboration can take various forms ranging from offering general advice and insights to active participation in a specific piece of research. Gatliff and Wendel (1998) confirmed that if two institutions are collaborating together they can have positive effects on teaching and learning. Collaboration in distance education is not an easy task when it deals with the human beings of different perceptions and institutions with varied and mixed interests. Researchers from different open distance institutions may collaborate by sharing data through correspondence or discussions at conferences, by visiting each other, or by discussions at conferences, by visiting each other, or by performing parts of a project separately and then integrating the results. There are benefits to institutional collaboration for open distance learning, which include among others increasing the skills of supervisors, providing broader knowledge for students and reducing costs.

This is supported by Bozeman and Corley (2004) when stating that some of the most frequent reasons for collaborative research are: access to expertise, access to unavailable equipment or resources, encouragement of multidisciplinary growth, improvement of the capability to get funds, prestige or visibility achievement, tacit knowledge acquirement about techniques, aggregation of knowledge for managing large and complex problems, productivity, education and training improvement of students and young researchers, increasing science specialization; or simply, the pleasure of working with 'colleagues. Furthermore Bozeman and Corley (2004) suggested that in many cases collaboration is the key mechanism for mentoring graduate students and postdoctoral researchers. According to Bozeman and Corley (2004) there are two levels of collaboration. The first level will be formed by students of different institutions and the second level will be formed by institutions that will jointly form the distance learning program together. Teaching and research collaboration between institutions in different institutions in the supervision of research students has become more important in recent years. One of the challenges facing open distance learning is supervision of students at a distance. The lecturers who supervise the postgraduate's student may share supervision with other lecturers from other institutions. This situation means that a single supervisor in a particular institution is unable to provide sufficient supervision for a student. Collaborative supervision by supervisors from different institutions is, therefore, appropriate and effective.

This was further expanded by Melin (2000) who stated that institutional collaborations are enhancing the productivity of individual student. There are significant advantages to collaboration, including the improved use of resources and better quality of supervision, achieved through collaboration with different experts. Co-supervision can be understood as pair of people working together to produce outcomes in which they are mutually involved. The cosupervisors or researchers from collaborative institutions share goals and common interests. Through their interactions they share knowledge, experience and skills. Normally when collaboration is formed by supervisors or institutions having diverse skills and experiences there are more resources to be involved in order to support their work and to reach their objectives. Human, intellectual and financial resources are the common resources that are involved when institutional collaborations are formed. For example, institutions can use their teaching and research resources more effectively and establish additional collaborative networks.

During institutional collaboration students can gain more research experience in and from different cultural and social contexts. Supervisors can benefit from a wider scope and richer experience in their student and project supervision. Many lecturers in open distance institutions believe that teaching and research collaboration in supervising research students is both, a necessary and useful (Melin, 2000). Due to globalisation and high numbers of students who are registering with $\mathrm{ODL}$ institutions more students and supervisors are looking for assistance from other institutions. They can conduct comparative studies about issues that are affecting different areas. This is supported by Melaville (1993) who look at collaboration as a series of interrelated activities undertaken by partners to address shared problems and achieve common goals. This kind of institutional collaboration can generate more comprehensive findings, together with more sustainable outcomes. It is also going to improve research output. Once the research output is increased then government will increase budgets for those institutions. Most of the universities have noticed that the resources that are provided to them by governments are insufficient so through collaborations universities will be able to share resources.

Institutions with more scientific instrumentations and well trained specialist can collaborate with those that are experiencing shortage of scientific resources. Once institutions collaborate they share resources such as modern telecommunications. The following communications technologies will be used: audio conferencing, audio graphic conferencing, computer conferencing and compressed video conferencing. According to Peters (2003) when more and more universities want to collaborate in the further development of distance education they find themselves faced with a dilemma. Either they are satisfied with fixing their teaching programmes onto media that cost little, and are sent to 
students which would be technically backward and a pedagogically minimalist solution, or they develop distanceeducation-oriented teaching materials and use modern telecommunications media, which would be technically abreast of the times and pedagogically ambitious but at the same time would exceed their financial and infrastructural possibilities.

When institutions collaborate it is going to increase the number of distance students, financing become easier and professional pedagogical course development and organisational coordination is possible by dividing tasks. Today in the world driven by intellectual property, there is an increased interest in collaboration in the area of research. Research can be considered as the activity of creating new knowledge. Though the goal of research is to create new knowledge, the purpose of research in each institution is to create new knowledge which other institutions can use to improve their teaching and learning (Gibbons, 1994).

Research helps collaborative institution to generate more revenue. When one institution collaborates with those that are technologically advanced they will push technology advances research that will bring more knowledge to the society. Collaboration can take different forms of ranging from offering general advice and insights to active participation in a specific piece of research. Researchers from collaborating institutions may collaborate by sharing data. Scholars who conceptualize learning as socially situated argue that group-based collaborative learning enables development of learning communities in the short term and communities of practice in the longer term.

\section{Theoretical Framework}

According to Moore (1991, pp. 1-6) as cited by Mafenya (2013, pp. 80-85), "Research that is not grounded in theory is wasteful." Against this back drop this study has made use of Moore's transactional distance theory. According to this theory there is a psychological separation between the student and the instructor, the student and the institution, student and student. This theory was regarded as the most suitable one for this study because of its ability to respond to the research question and the research objectives. Most recently, Moore has described it as: "The transaction that we call distance education occurs between individuals who are teachers and learners, in an environment that has the special characteristic of separation of one from another, and a consequent set of special teaching and learning behaviours. It is the physical separation that leads to a psychological and communications gap, a space of potential misunderstanding between inputs of instructor and those of the learner, and this is the transactional distance." (Moore, 1991, pp. 1-6). However, this psychological separation could be narrowed or bridged through the use of technologies to increase institutional collaboration.

\section{Research Methodology}

The methodological approach for this study is qualitative research design. According to McMillan and Schumacher (2006, 315) qualitative research is "an inquiry in which researchers collect data in face-to-face situation by interacting with the selected persons in their setting (e.g. field of research). Qualitative research describes and analyses people's individual and collective social actions, beliefs, thoughts and perceptions. The research question "How does institutional collaboration increase research output in open distance learning?" Provided the focus for this study. To find answers to the research questions data were collected using semi-structured interviews to find the lecturers' experiences concerning institutional research collaboration. The sample for this study came from two universities namely: the University of South Africa, a dedicated distance learning university and the University of Johannesburg, a dual mode university. Data were collected from academics from the following four colleges namely: College of Education, College of Human Sciences, College of Economics and Management Sciences, College of Agriculture and Environmental Sciences. Eight academics were purposefully selected to participate in this study. Data were digitally recorded, transcribed and analysed using Collaizi's (1978) framework. This framework was utilised because of its ability to provide steps of data analysis. The following stages were employed to analyse data:

- Step one: The researcher read and re-read the transcribed-word-for-word interviews to make sense of them and acquire a feel for each description

- Step two: The researcher extracted significant statements, which pertained directly to the proposed phenomenon, from the description

- Step three: The researcher formulated meanings after analysing each significant statement. The researcher again re-read the original protocols to make sure that the original description was captured in the extracted significant statement meanings

- Step four: The researcher organised the formulated meanings into clusters, which allowed themes to emerge. 
The researcher again referred to the original protocol for validation, being cognisant of repetitive themes and discrepancies

- Step five: The researcher integrated themes into an exhaustive description.

- Step six: The researcher made a concise statement of the exhaustive descriptions and provided a fundamental statement of identification. In order to understand students' experiences the researcher had to move from individual phenomenological structure to the general description of situated structures.

It is through this process that several themes were identified from each participant and then clustered into a number of general themes that appeared to be common to all the participants' descriptions. The final step in data analysis involved an interpretation or meaning of data, and it was at this stage that the clustered themes and meanings were used to develop the textural descriptions of the experiences. From the textural and structural description an integration of the meanings and essences of the phenomenon were constructed. This included making use of the verbatim examples from the transcribed interviews.

- Step seven: The researcher presented a concise statement of the exhaustive description to the original participants of the study in order to verify the statement.

\section{Discussion of the Study Findings}

The purpose of this study was to explore and describe how institutional collaboration and research training can be utilised to increase research output. On the basis of the data collected, recorded and analysed the study revealed the following important themes: Workload, time constraint, teamwork, lack of resources and lack of experience. The researcher has therefore discussed the study findings as per themes that were revealed during the interviews:

\subsection{Workload}

The first theme from the data indicates that they have a problem of having a huge workload. This theme indicated that most of the lecturers were experiencing problems when it came to workload especially when it is time to write tutorial and examinations. The study discovered that there is a huge workload for them. One of the lecturers commented by saying: "We are heavily pressed as we are expected to have more research output while on the other hand we are faced with marking and writing tutorial letters and study guides."

\subsection{Time constraint}

This theme on time constrain was deliberated in full by all the participants. One of the participants had this to say concerning institutional collaboration "I am expected to attend meetings, workshops, and other activities that are taking place on daily basis". Time constraints and workload were the two issues that lecturers complained about. Lecturers felt that they had little time as they are expected to attend meetings, workshops, seminars and to write tutorial letters whereas on the other hand had to give feedback to students. They complained that already they are supervising more than three students and when they had to supervise students from other universities it is difficult for them. Lecturers from collaborating institutions end up being able to share and to accommodate each other even if it was not immediate. Lecturers from technologically advanced institutions end up sharing more knowledge to peers who were not used to that end up being matured into that field.

\subsection{Teamwork}

Lecturers who participated in collaboration expressed significantly greater overall satisfaction about their learning experience. They also showed greater satisfaction with regard to working and socially interaction with lecturers and students from different universities. The institutions that were not technologically advanced and given chance to collaborate with technologically advanced institutions were able to conduct research easily. They commented that team members from other collaborated universities were excellent teammates. Lecturers thought that co-supervision was a good idea that facilitated lecturer's collaborations, as well as allowed them an opportunity to gain new skills and study new topic in greater depth. Lecturers who were not used to co-supervising complained that it was not easy to guide students during co-supervision as they sometimes give opposing feedback. One of the academics had this to say: "Student supervision needs a lot of dedication from all parties because when you work as a team it makes one's job 
easier." From the above statement one can conclude that collaboration is important since it involves a lot of sharing ideas and fosters team spirit.

\subsection{Lack of resources}

The institutions that do not have enough resources benefit a lot through collaboration. Lecturers who participated in interviews expressed the importance of collaborating with institutions that have adequate resources. The issue of resources is affecting most of the institutions since it is highly unlikely that ODL institutions could share their resources with conventional institutions. On asking how the issue of resources could be dealt with, some of the interviewed participants had this to say: "Our governments should enforce policies and procedures that should encourage the building of adequate research infrastructure. It is important to provide resources that will make it possible for researchers to collaborate." The above quotation indicates that the government and the private sector should collaborate in the provision of resources.

\subsection{Lack of experience}

In this theme lecturers expressed various opinions with regard to lack of experience. Lecturers thought that the cosupervision was a good idea. It allows them an opportunity to gain new skills and even to study new topic in greater depth. One of them had this to say: "I have been working here for two years, what I experienced is that three masters students were allocated to me. I must supervise and guide them even if I do not have experience. Due to lack of experience it is impossible to guide them properly". The universities and departments can form stronger alliances or teams for both teaching and research through collaboration. They can gain experience and support for exploring and developing new teaching and research directions. Supervisors can gain wider scope and richer experience in research and project supervision. Supervisors can gain more tangible research outputs, including publications, which also generates increased opportunities and greater incentives for supervisors to be more active in research. Students can benefit from research experience in different cultural and social contexts. Students will be able to interact with supervisors and students from different social cultural and environmental contexts. For example postdoctoral students can add more value to the research of the other institutions. Students can explore and form collaborative networks with their supervisors and students in other universities. Through institutional collaborations students themselves often motivate and help others and advise them about opportunities that are available for them.

\section{Implications for the Study}

Institutional collaboration in supervising research students has an important role for research training, the development and achievement of design research. All stake-holders can benefit from joint supervision. It can destroy the original goals and generate negative results. For example, without carefully identified roles and pre-agreed sharing of credit among supervisors, joint supervision may lead to bad relationships and disagreements, not only among supervisors and students but at departmental and university level. The partners must feel that their interests and purpose collaboration should be "equal status for all." The partners must feel that their interests and purpose for entering the collaboration are well served, and should be able to see actual returns-whether through increased enrolment or improved infrastructure and services - on their investment in the partnership. There is a widespread wish for institutional collaboration and research training, and longer stays in advanced and well-resourced institutions are considered an important means of achieving high research output.

Despite the difficulties which we have noted, most interviewees were positive and regarded the research output benefits as great. Nevertheless, it is still only the minority of doctoral degree students and supervisors who have experienced co-supervision as confusing. Institutional factors such as economic aspects and work load, academic matters (e.g. the topic of a thesis), and personal conditions (family obligations) are reasons why more have not done so. There are other ways of institutionalising collaborative research training, of which participation in summer schools, conferences, workshops, etc was often noted. These too can allow researchers at an early stage of their careers to build up the personal networks within the scientific community which may be so important to their subsequent professional development. 


\section{Conclusion}

This study has revealed that institutions need to work as a team to advance knowledge. They do this by planning together the research activities. Through collaboration institutions can invite experts to come and train others, organise workshops, seminars, conferences aimed at equipping other staff members so that they become experts. Local institutions can collaborate with international institutions, when they collaborate with them they can engage in joint designs, joint research and developmental projects, joint investigations and also student exchange programmes. Through international collaboration institutions will have an access to international funds that are used to improve their practice guidelines. Institutions can use those funds to carry out research and development projects. They can also invite well-known international researchers to come and train novices on exchange programmes. This kind of collaboration should be done by students, members of the teaching and administration staff. The reason behind this collaboration is to bring about a culture of improving institutional teaching and learning strategies. If institutions collaborate they will end up becoming comprehensive research oriented institutions.

\section{Reference}

Austin, A.E. \& Baldwin, R.G. (1992). Faculty collaboration: Enhancing the quality of scholar-ship and teaching. Washington, DC: ERIC Clearinghouse on Higher Education

Bardach, E. (1998). Getting Agencies to Work Together: The Practice and Theory of Managerial Craftsmanship. Washington, DC: Brookings Institution Press

Bozeman, B. \& Corley, E. (2004). Scientists' Collaboration Strategies: Implications for Scientific and Technical Human Capital. Research Policy, 33(4), pp. 599-616.

Collaizzi, P. (1978). Psychological research as the phenomenologist views it. New York: Oxford University Press

Fox, M.F. \& Faver, C.A. (1982). The process of collaboration in scholarly research. Scholarly for Publishing, 13, pp. 327-339.

Gatliff, B. \& Wendel, F.C. (1998). Inter-institutional collaboration and team teaching. American Journal of Distance Education, 12 (10), pp. 26-37.

Gibbons, M. (1994). The New Production of Knowledge: The Dynamics of Science and research in Contemporary Societies. London: Sage Publishers.

Gray, B. (1989). Collaboration: Finding Common ground for Multiparty Problems. San Francisco: Josey-Bass

Gros, B. \& Adrian, M. (2005). The use of virtual forums to promote collaborative learning in higher education. Educational Technology.45 (5), pp. $17-20$.

Hathorne, L.G. \& Ingram, A.L. (2002). Online collaboration: Making it work. Educational Technology, 42 (1), pp. 33-40.

Hemmings, M.B. (1984). Next steps in public-private partnerships. Syracuse, NY: ERIC Clearinghouse on Information Resources.

Kitchen, D. \& McDougall, D. (1999). Collaborative Learning on the Internet. Journal of Educational Technology Systems, 27 (3), pp. $245-58$.

Kyvik, S. \& Larsen, I.M. (1997). The exchange of knowledge. A small country in the international research community. Science Communication, 18, pp. 238-264.

Mafenya, N.P. (2013). Enhancing student success through engagement in assessment practices in open and distance learning: A phenomenological approach. In Education Across Space and Time-meeting the diverse needs of the distance learner, edited by R. Sims and M. Kigotho, 80-94. ODLAA: Australia.

Mattessich, P.M. (2001). Collaboration: What Makes It Work. St. Paul, Minnesota :Amherst H.Wilder Foundation.

McCord, R. S. (2002). Breaking the School District Boundaries: Collaboration and Cooperation for Success, Education, pp. 389-389

McMillan, J. \& Schumacher, S. (2006). Research in Education. New York: Allyn \& Bacon

Melin, G. (2000). Pragmatism and Self-organization: Research Collaboration on the Individual Level, Research Policy, 29 (1), pp. 31-40

Mellaville, A.M. (1993). Together We Can: A Guide for Crafting a Pro-family System of Education and Human Services. Washington: United States Government Printing.

Moore, M.G. (1991). Distance Education Theory. The American Journal of Distance Education, 5(3), pp.1-6.

Peters, O. (1993). Distance Education in post-industrial society. New York: Routledge

Zuckerman, H.S.; Kaluzny A.D. \& Rickets,T.C.(1995). Alliances in Health Care Management Review, 20, pp.54-56 